\title{
Using Computerized Maintenance Management System (CMMS) in Healthcare Equipment Maintenance Operations
}

\author{
Mohammad Lafi Alburaiesi \\ Ministry of Public Works-Kuwait, Kuwait
}

Received: 06/06/2020

Accepted: 03/09/2020

Published: 20/12/2020

\begin{abstract}
Healthcare and medical equipment sustainability is very important, we need to have an efficient and high performance devices. If such systems or devices are fail every time this will effect patients and the medical system at all. Healthcare equipments like ventilators and other devices nowadays has a good priority and important because of Covid19 which effects all countries now. Since these devices exposed some defects which will effect on its role it is very important to study and analyze such defects, classify them and how to apply CMMS in such maintenance operations. This paper aims to use CMMS in maintenance operations of equipments in healthcare sector. Also a classification of failures of such equipments is generated to be an input for CMMS programs. The second thing is to use Equipment maintenance (EM) number in classifying such devices to be ready for using CMMS. A sample of medical devices are studied and the equipment management number (EM) is calculated here for some selected devices if it reaches some specific value it needs maintenance and CMMS will manage the maintenance operations required. CMMS procedures, transactions and benefits are discussed in this paper. It is found that in the first step we should determine the devices included in inspection and maintenance operations by using EM number, then specifying the maintenance period and inspection frequency after that following some specific steps to manage the maintenance operations followed by CMMS programs. Equipment maintenance (EM) number is very important to determine whether the equipment need maintenance or not, and it also determine the inspection and maintenance duration and so such devices be ready to be used all the time. CMMS can be efficiently used in maintenance operations of healthcare equipments. The use of CMMS software or application is used in processing entered data of devices or systems following the commands step-by-step to construct a CMMS system for each plant or system, this will simplify the monitoring and inspections and maintenance operations which increasing availability of the device and decreasing downtime
\end{abstract}

Keywords: Healthcare, Medical devices, Equipment Management Number, CMMS

\section{Introduction}

Medical equipments are a significant and essential segment of all human services frameworks. They are presented and used for persistent conclusion and treatment. These clinical gadgets must be guarded in condition so as to forestall wounds in patients and staff [1-3]. As indicated by the World Health Organization, in the USA in 2010 roughly 412 US dollars were allocated to clinical gear upkeep [4]. Subsequently, support the board framework is basic to improve the unwavering quality of clinical hardware and altogether improve security and costproductivity [5]. For instance, appropriate upkeep can build the life of hardware. Likewise, this procedure guarantees us about giving proper wellbeing administrations and sparing the rare assets. In any case, numerous medical clinics and medicinal services associations don't profit by upkeep greatness [6]. Clinical Equipment Management System (MEMS) incorporates the hardware stock, a work request framework, the preventive support plans/methodology, re-appropriating contract the executives and all assistance history records. Today, the Clinical and Biomedical Engineering (CE/BME) is answerable for the social insurance resource the executives and human services innovation evaluation, clinical staff wellbeing, fix and upkeep, hazard and security the board, and furthermore differentiate checking and quality improvement [1-3].
Furthermore, Computerized Maintenance Management Systems (CMMS) is a key data asset in most medicinal services frameworks to follow each bit of hardware and keep up precise records of stock and information for clinical gear [5, 7, 8]. There are two most common approaches for medical equipment maintenance that includes preventive and the corrective strategies. Preventative Maintenance (PM) is a scheduled process of ensuring medical devices; and the equipment are kept in perfect working conditions. Corrective Maintenance $(\mathrm{CM})$ is repairing and restoration of medical devices that failed to function. These strategies help hospitals to assess or improve maintenance, quality and performance. At this point, hospitals should be considering their capability and situation to apply best maintenance systems for the large number of different medical devices in the equipment management processes. Healthcare organizations should determine the criteria to assess their medical equipment maintenance management. There is a lack of specific and precise tool to assess the process of medical equipment maintenance management in Iranian hospitals. Hence, we extracted the factors affecting the medical equipment maintenance management through a systematic review method [9].

A CMMS is a software package that contains a PC database of data about an association's support activities. In HTM, the 
CMMS is utilized to computerize the documentation of all exercises identifying with clinical gadgets, including gear arranging, stock administration, restorative and preventive upkeep methodology, save parts control, administration agreements, and clinical gadget reviews and cautions. The gathered information can be broke down and utilized for innovation the executives, quality affirmation, work request control and planning of clinical gadgets (3). The choice to robotize a HTM framework or supplant a current CMMS relies upon the individual conditions of the wellbeing office, including working systems, data innovation (IT) foundation and accessible spending plan. So as to viably aid the administration and upkeep of clinical hardware, a CMMS should exhaustively address the issues of the client. Albeit significant merchants endeavor to build up a framework that all around addresses the issues of all HTM chiefs, no accessible framework presents a total arrangement. Most, be that as it may, can be altered to meet the particular needs of the wellbeing office. On the other hand, an IT firm can be contracted to build up a CMMS bundle customized to nearby necessities. A redid CMMS bundle is commonly increasingly costly yet on the off chance that very much planned and kept up will regularly deliver a progressively acceptable arrangement that addresses nearby issue. A CMMS can be utilized to: (a) institutionalize and blend data inside a HTM Program; (b) aid the arranging and observing of investigation and preventive upkeep, and calendar and track fixes; (c) screen gear execution pointers, for example, mean time between disappointments, down time and upkeep costs for individual or hardware gatherings of a similar model, type or maker; (d) screen clinical building staff execution markers, for example, rehashed fixes by a similar staff part for a similar issue, normal vacation related with people, and beneficial work time for people or gatherings; (e) create reports that can be utilized to design client preparing programs dependent on hardware disappointment inclines in specific divisions or wellbeing offices; (f) have libraries of administrative necessities and wellbeing data; $(\mathrm{g})$ create the proper documentation for accreditation by administrative and standard associations; (h) create reports to aid the checking and improvement of the [10]. Figure 1 shows CMMS implementation flow chart

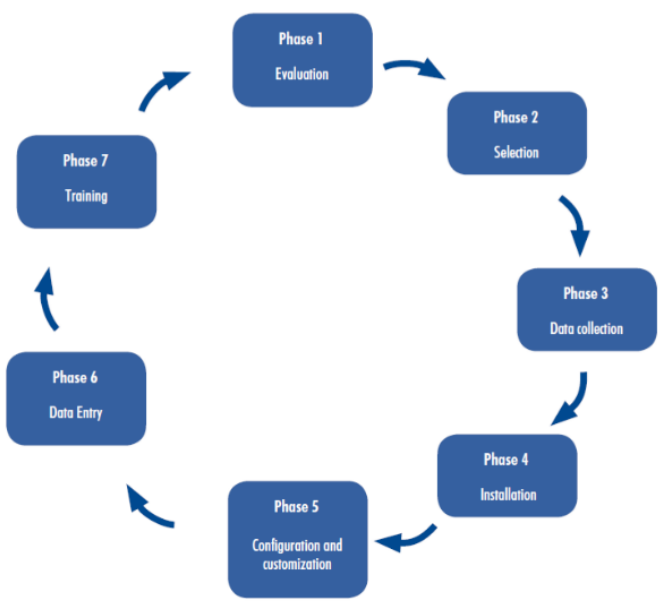

Figure 1: CMMS implementation flow chart [10]

\section{Factors affecting equipment failures}

When investigating an unexplained disappointment, ecological components ought to be taken into due thought. For instance, clinical gadgets that require electrical force might be unfavorably influenced by power issues. Preferably, electrical force ought to have a consistent voltage (of the proper worth); be liberated from transient twists, for example, voltage spikes, floods or dropouts; and be dependable, with just uncommon loss of intensity. Shockingly, these perfect qualities don't generally exist in many creating nations. Specialized work force ought to team up with those liable for the electrical force framework in the human services association to help make the framework work as adequately as could reasonably be expected. This may incorporate buying voltage controllers, introducing uninterruptable force supplies (UPS), utilizing flood defenders, and abstaining from associating expansion drives/plug sheets in arrangement. Moreover, specialized faculty should work with the office staff to guarantee that a useful back-up generator is set up and that the change to helper power is made in less than 10 seconds. Another option might be to choose and buy gear that is battery worked. When thinking about obtaining new hardware, it is additionally significant for the specialized staff to guarantee that the electrical force framework is equipped for supporting it. At the point when it can't, it frequently bodes well to decide on not so much advanced but rather stronger equipment [11].

Similarly, technical personnel ought to know about how clinical gadgets interface with other utility frameworks (for example clinical gas and vacuum frameworks, temperature control and ventilation frameworks, water supply, data innovation and correspondence foundation, and so forth.). Also, by and by, they ought to team up with others in the association to improve the capacity of the utility frameworks to help clinical gear. One of a kind parts of the physical condition, for example, high temperature and dampness, can antagonistically influence clinical hardware intended for use in mild atmospheres or controlled situations. Upkeep strategies in a specific nation or district may should be balanced dependent on these nearby factors. The age and state of the human services office may likewise assume a job in clinical gear disappointment. After some time, utilities frameworks will debase and may get over-burden and additionally obsolete. More seasoned offices will have been worked to more established measures. Indeed, even new offices may not fulfill every single material rule. Along these lines, it is regularly important to test the utility foundation as opposed to expect it is working suitably [11].

Computerized Maintenance Management Systems are bundled programming apparatuses structured explicitly to help organizations and support frameworks in upkeep the board. Most organizations that keep up gear today have a CMMS being used and there are many economically accessible bundles to look over. The development and advancement in IT has prompted an emotional increment in the ability and accessibility of programming instruments to help upkeep. Normally, littler organizations utilizing particular arrangements are generally happy with their CMMSs. The two most significant highlights for fruitful usage are simplicity of adjusting to upkeep procedures and ease of use [12]. CMMS offers powerlessness of conveying helpful data and ready to fathom the offices requests over the need support plan. The essential data, for example, side effect, issue, restorative activity and unordinary conditions will be refreshed the significant authentic information for future investigating endeavors. In this manner, the new framework can play out the best choice and consequent activity precisely [13]. CMMS can be utilized to deal with all planned protection support and on request work orders. Like all CMMS programs, both protection and on request work orders are booked and followed inside the framework. Areas, structures, gear, and vehicles are recognized, stocked, and connected with all work orders. Routinely planned safeguard upkeep (PM's) work orders are consequently created and are appointed to support specialists 
dependent on criteria gave by the office chiefs. Request work orders are commonly one-time occasions, for example, fixes and produced by staff dependent upon the situation. A work request stays open until shut by the appointed professional hence giving responsibility. Work request history, support patterns, costs, stock, and key execution pointers are observed and checked on by utilization of continuous reports [14].

CMMS is presently reasonable and an unquestionable requirement have innovation stage for proprietors and occupiers of land coming about in: (a) increased inhabitant fulfillment through Internet-based work request demands; (c) procurement of administrations that gives serious offering to outsider administrations, to incorporate little and capital undertaking conveyance; (d) owner certainty that their advantage is being figured out how to the best quality of care and now how does CMMS vary from customary property and office the board? (e) facility/property the board staff doesn't approach innovation apparatuses and preparing that expands profitability and disposes of re-work; (g) computerized upkeep the executives frameworks (CMMS) are commonly exclusive as well as permit expenses restrict use for medium to little structure proprietors; (h) our innovation is worked around lean work processes that diminishes the requirement for director commitment and speeds up administration conveyance; (i) vendor the executives is incorporated with the innovation that screens work request reaction times, expanding occupant fulfillment versus trusting that a supervisor will finish the solicitation $(\mathrm{g})$ financial controls help diminish working costs and guarantee merchant work demands are finished inside spending plan [15].

Computerized Mechanized Maintenance Management Systems-CMMS will defeat such issues and offers a quick reaction observing framework, ongoing crisis support framework for machines and types of gear and solid and precise framework for disappointment checking of types of gear. CMMS are utilized for all parts of support arranging, the executives and control. CMMS must be adaptable and versatile, in light of the fact that each firm is viewed as extraordinary. CMMS is a product bundle intended to oversee high volumes of advantage data, it emphatically underpins the accompanying: Agency strategies and methods, Equipment information, Work request control, Preventative upkeep rehearses, lastly Materials control [16, 23]. Another meaning of Computerized Maintenance Management System (CMMS) is a product item that assist organizations with running their upkeep tasks proficiently. With these frameworks, organizations can design preventive upkeep, oversee remedial support, track machine hours, tires and different consumables, screen guarantee periods, track work and fuel costs and oversee stock. Further developed CMMS frameworks incorporate likewise with other organization capacities, for example, venture arranging, resourcing, and bookkeeping [17,22, and 24]. Clinical types of equipments are a significant and essential segment of all human services frameworks. They are presented and used for medical persistent determination and treatment. These clinical gadgets must be guarded in condition so as to forestall wounds in patients and staff [19, 20, and 21].

\section{Methodology}

\subsection{Developed Model}

For Risk-based biomedical hardware, the board program is created Equipment consideration criteria have been created to assess each bit of gear being used at a medical clinic or wellbeing office. The accompanying subtleties an adjusted variant of the Smith model (see reference 6) where a numerical worth has been relegated to every gadget type by ordering its gear work, clinical application and required upkeep. Including the number from every subgroup and including or subtracting a factor dependent on hardware disappointment history yields the equipment management (EM) number.

EM \# = Function \# + Application \# + Maintenance \# + History \# (1)

To such an extent that all gadgets with an all-out EM number of at least 12 will be remembered for the Program and planned for investigations and preventive upkeep. During the acknowledgment testing, any new gadget will be remembered for the Program if the gadget has been recently assessed and order for incorporation. In the event that the gadget has not been recently assessed, another gadget grouping will be made. It will be assessed by the delineated technique to create an EM number and will be remembered for the program if suitable. Whenever included, a presentation affirmation assessment and preventive upkeep strategy will be composed for the new gadget [18]. Function scores can be calculated by Table 1 . Application scores can be calculated by Table 2. Maintenance scores can be calculated by Table 3 . History scores can be calculated by Table 4 .

Table 1: Function scores

\begin{tabular}{|l|l|c|}
\hline Category & Function description & Point score \\
\hline \multirow{3}{*}{ Therapeutic } & Life support & 10 \\
\cline { 2 - 3 } & Surgical and intensive care & 9 \\
\cline { 2 - 3 } & Physical therapy and treatment & 8 \\
\hline \multirow{3}{*}{ Diagnostic } & Surgical and intensive care monitoring & 7 \\
\cline { 2 - 3 } & Additional physiological monitoring and diagnostic & 6 \\
\hline Analyical & Analytical laboratory & 5 \\
\cline { 2 - 3 } & Laboratory accessories & 4 \\
\cline { 2 - 3 } & Computers and related & 3 \\
\hline Missellaneous & Patient related and other & 2 \\
\hline
\end{tabular}

Table 2: Application scores

\begin{tabular}{|l|c|}
\hline Description of use risk & Point score \\
\hline Potential patient death & 5 \\
\hline Potential patient or operator injury & 4 \\
\hline Inappropriate therapy or misdiagnosis & 3 \\
\hline Equipment damage & 2 \\
\hline No significant identified risk & 1 \\
\hline
\end{tabular}

Table 3: Maintenance scores

\begin{tabular}{|l|c|}
\hline Maintenance requirement & Point score \\
\hline Extensive: routine calibration and part replacement required & 5 \\
\hline Above-average & 4 \\
\hline Average: performance verification and safety testing & 3 \\
\hline Below-average & 2 \\
\hline Minimal: visual inspection & 1 \\
\hline
\end{tabular}

\subsection{Maintenance interval}

The maintenance requirement values are also used to determine the interval between each inspection and maintenance procedure for each device type. All devices classified as extensive (characteristic value of 4 or 5) are given a preventive maintenance interval of six months. While Devices with average or minimal requirements (values of 3,2 or 1) are scheduled for preventive maintenance annually. On the other hand, Devices with an EM number of 15 or above will 
be scheduled for inspection at least every six months. Finally, Devices with an EM number of 19 or 20 will be given an inspection interval of four months [18].

Table 4: History scores

\begin{tabular}{|l|c|}
\hline Average equipment failures & Factor \\
\hline Significant: more than one every 6 months & +2 \\
\hline Moderate: one every 6-9 months & +1 \\
\hline Average: one every 9-18 months & 0 \\
\hline Minimal: one every 18-30 months & -1 \\
\hline Insignificant: less than one in the past 30 months & -2 \\
\hline
\end{tabular}

\section{Results and Discussion}

\subsection{Devices not included in the program}

All patient care-related equipment including therapeutic, monitoring, diagnostic or analytical equipment not included in the program, because it did not receive an EM number of 12 or above, may still be included in the hospital's biomedical equipment inventory and be covered on a repair-only basis
[18]. Table 5 shows the data of some devices (collected from literature) which will be used to calculate the EM number and then classify the devices under considerations. After determine the devices that need a maintenance operations we should construct a CMMS model for such devices by the following order: in Step one inter to the CMMS software and inter the data required for the two sites, Figure 3 shows the main screen of the CMMS software. The data entered to CMMS software including four main parts. In step two assigning specification for each item. Each item be maintained has a specification includes: its maintenance requirements, dates and its priority and other information. In step three constructing work orders for each item. Using CMMS SW maintenance work orders can be assigned for each item. Then finally the CMMS can give a summary for the device operating and downtimes during a specific period. Figure 4 shows a sample of such summary.

The results show that all operating devices working in healthcare systems should be ready to use in all healthcare situation specially in emergency cases, the EM number is a good tool to specify the need of the device to be inspected, and also maintained and what are required to be ready in use. And also it determine the frequency of inspections.

Table 5 EM\# for some selected devices

\begin{tabular}{|l|l|l|l|l|l|l|l|}
\hline Device & $\begin{array}{l}\text { Eq. } \\
\text { Function }\end{array}$ & $\begin{array}{l}\text { Eq. } \\
\text { Application }\end{array}$ & $\begin{array}{l}\text { Eq. } \\
\text { Maintenance }\end{array}$ & $\begin{array}{l}\text { Eq. } \\
\text { History }\end{array}$ & $\begin{array}{l}\text { EM\# } \\
\text { frequency }\end{array}$ & Class \\
\hline Cardiac Output Comp. & 7 & 3 & 2 & 0 & 12 & $\mathrm{I}$ \\
\hline Blood Warmer & 8 & 3 & 2 & -2 & 11 & $\mathrm{~N}$ \\
\hline Fetal Monitor & 7 & 3 & 3 & 0 & 13 & $\mathrm{I}$ & $\mathrm{A}$ \\
\hline Humidifier & 8 & 3 & 3 & 1 & 15 & $\mathrm{I}$ \\
\hline Light source & 7 & 3 & 3 & -2 & 11 & $\mathrm{~N}$ & - \\
\hline Microscope & 6 & 3 & 3 & -2 & 10 & $\mathrm{~N}$ \\
\hline
\end{tabular}

I: Included, N: Not included, A: Annual, T: Three-yearly, S: Semi-annual

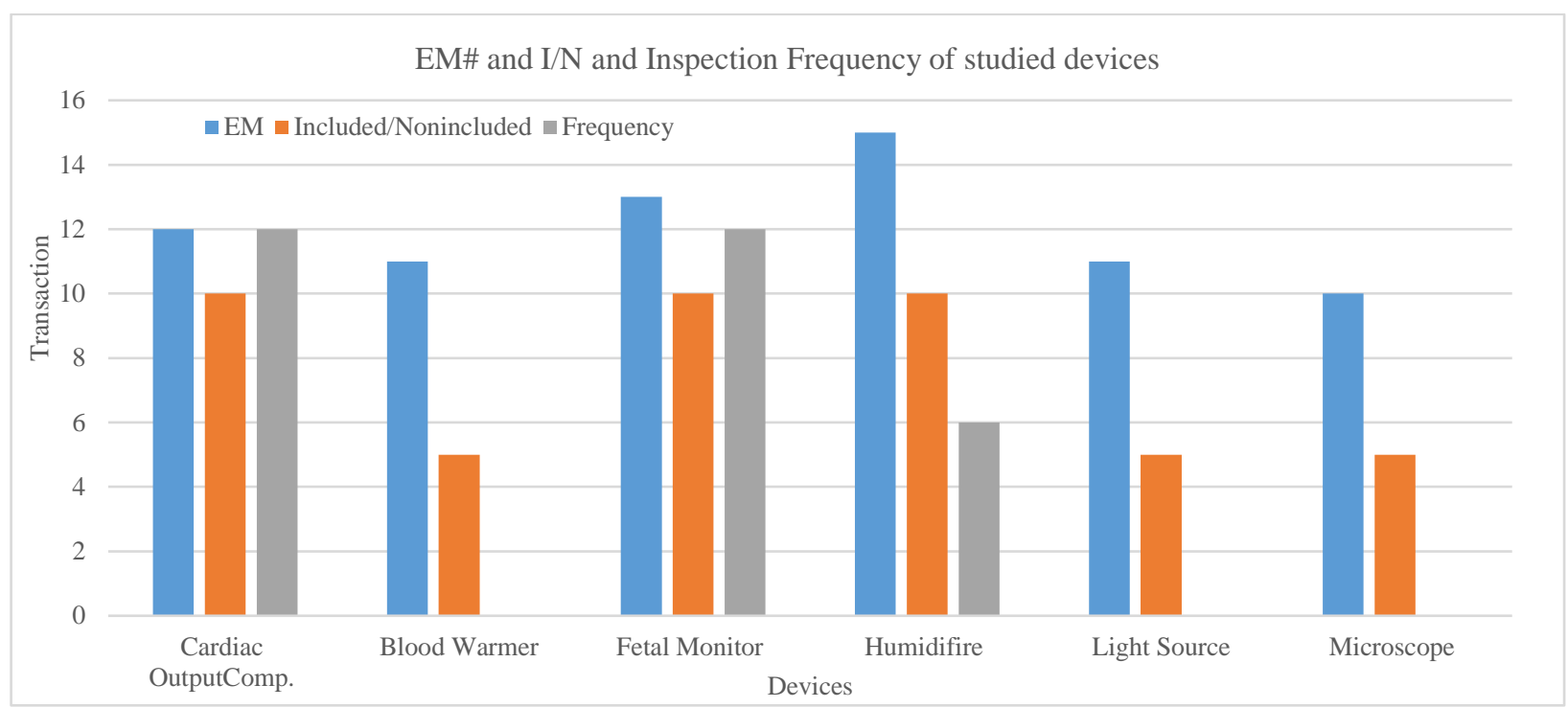

Figure 2: Equipment Maintenance Number and status and transactions for some studied devices 


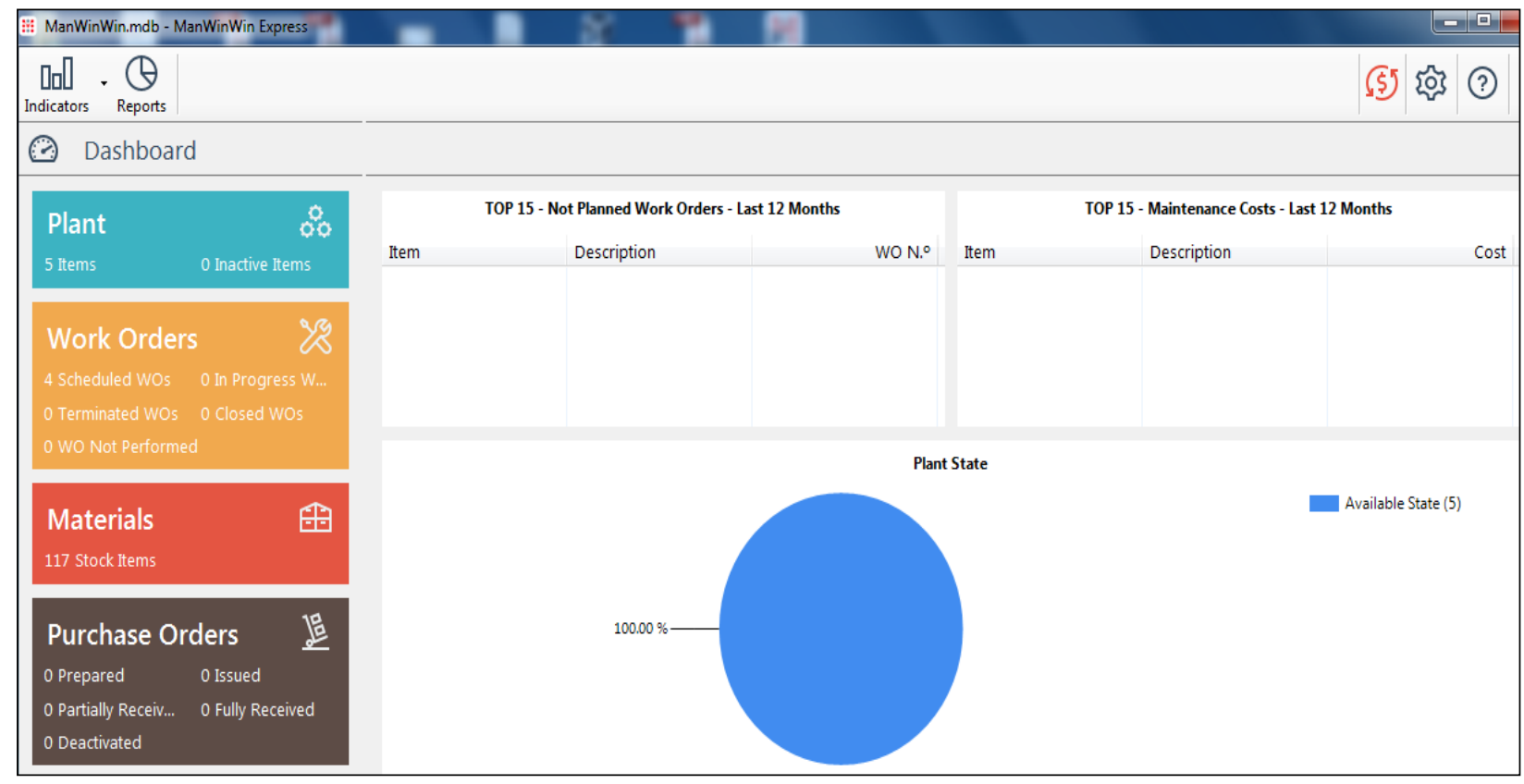

Figure 3: Main screen of CMMS

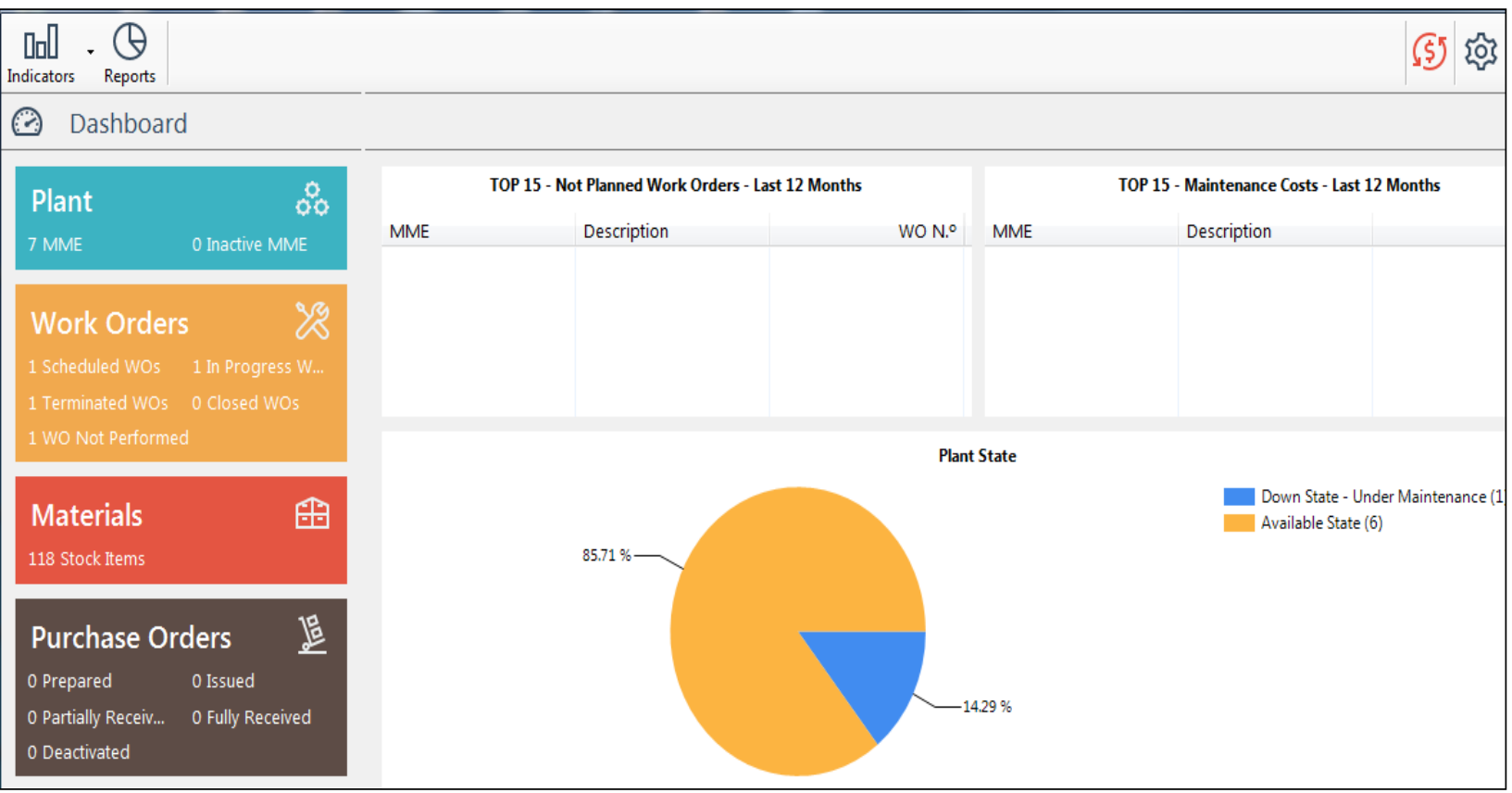

Figure 4: Summary of availability state and downtime of the device

\section{Conclusions}

This paper discussed application of CMMS on healthcare and medical devices maintenance systems. The first step is to know or assess which devices need maintenance by determine equipment maintenance number which is used to classify the device if it needs maintenance or not. Then using some CMMS software or application in entering data of devices or systems and by following the commands step-by-step to construct a CMMS system for each plant or system, this will simplify the monitoring and inspections and maintenance operations which increasing availability of the device and decreasing downtime.

\section{Ethical issue}

Authors are aware of, and comply with, best practice in publication ethics specifically with regard to authorship (avoidance of guest authorship), dual submission, manipulation of figures, competing interests and compliance with policies on research ethics. Authors adhere to publication requirements that submitted work is original and has not been published elsewhere in any language.

\section{Competing interests}

The authors declare that there is no conflict of interest that would prejudice the impartiality of this scientific work.

\section{Authors' contribution}

All authors of this study have a complete contribution for data collection, data analyses and manuscript writing. 


\section{References}

[1] Lenel A, Temple-Bird C, Kawohl W, Kaur M. How to organize a system of healthcare technology management. Geneva: World Health Organization. 2005.

[2] David Y, Jahnke EG. Planning hospital medical technology management. IEEE Eng Med Biol Mag. 2004;23(3):73-79.

[3] David Y, Judd TM. Management and Assessment of Medical Technology, Clinical Engineering (Principles and Applications in Engineering). CRC, New York. 2003.

[4] Pammolli F, Riccaboni M, Oglialoro C, Magazzini L, Baio G, Salerno N. Medical devices competitiveness and impact on public health expenditure. 2005.

[5] Hamdi N, Oweis R, Abu Zraiq H, Abu Sammour D. An intelligent healthcare management system: a new approach in work-order prioritization for medical equipment maintenance requests. J Med Syst. 2012;36(2):557-67.

[6] Ginsburg G. Human factors engineering: A tool for medical device evaluation in hospital procurement decision-making. J. Biomed. Inform. 2005;38(3):213-9.

[7] Wang ZH. Application Research on the Internet in the Management of Medical Equipment Maintenance. Applied Mechanics and Materials. Trans Tech Publ. 2014;651:1535-1538.

[8] Basiony M. Computerized equipment management system. J Clin Eng. 2013;38:178-84.

[9] Bahreini R, Doshmangir L, Imani A. Affecting Medical Equipment Maintenance Management: A Systematic Review. Journal of Clinical \& Diagnostic Research. 2018 Apr 1;12(4)

[10] WHO. Computerized maintenance management system, WHO Medical device technical series report. Switzerland.

[11] Mukattash A, Fouad RH, Kitan H, Samhouri M. Computer-Aided Maintenance Planning System for Industrial Companies. Jordan Journal of Mechanical \& Industrial Engineering. 2011 Jun 1;5(3).

[12] Laurila J. Developing Computerized Maintenance Management System. Master thesis. Helsinki Metropolia University of Applied Sciences. 2017

[13] Ismail ZA, Kasim N. Improving maintenance management practices on conventional method at Malaysian polytechnic. 2012.

[14] Port of new port. Facilities Maintenance \& Operations Plan. 2016. www.portofnewport.com.

[15] Norris J. Computerized maintenance management systems (CMMS). Indianapolis, IN 46240. 2017. www.colliers.com.

[16] Jafarnejad A, Soufi M, Bayati A. Prioritizing Critical Barriers of Computerized Maintenance Management System (CMMS) by Fuzzy Multi Attribute Decision Making (F-MADM)(Using LFPP). Kuwait Chapter of the Arabian Journal of Business and Management Review. 2014 Nov 1;4(3):11.

[17] Mutia D, Kihiu J, Maranga S. Developing an In-house Computerized Maintenance Management System for Hospitals. Industrial Engineering Letters. 2012;2(3):1-9.

[18] WHO. Medical equipment maintenance program overview, WHO Medical device technical series. Switzerland

[19] Lenel A, Temple-Bird C, Kawohl W, Kaur M. How to organize a system of [1] healthcare technology management. Geneva: World Health Organization. 2005.

[20] David Y, Jahnke EG. Planning hospital medical technology management. IEEE [2]Eng Med Biol Mag. 2004;23(3):73-79.

[21] David Y, Judd TM. Management and Assessment of Medical Technology, [3]Clinical Engineering (Principles and Applications in Engineering). CRC, New York. 2003.

[22] Ahmad I, Ahmad S. The Mediation Effect Of Strategic Planning On The Relationship Between Business Skills And Firm's Performance: Evidence From Medium Enterprises in Punjab, Pakistan. Opcion. 2019;35(24):746-78.

[23] Ahmad I, Ahmad S. Multiple Skills and Medium Enterprises' Performance in Punjab Pakistan: A Pilot Study. Journal of Social Sciences Research. 2018;Special, (4):44-9.

[24] Ahmad, I., Sahar. Waste Management Analysis From Economic Environment Sustainability Perspective. International Journal Of Scientific \& Technology Research. 2019; 8(12), 1540-1543. 